\title{
El etnosimbolismo de Anthony D. Smith y los orígenes de la nación armenia
}

\author{
Ricardo Torres*
}

\section{Resumen}

Para Smith, uno de los teóricos del etnosimbolismo, naciones y nacionalismo sólo pueden ser entendidos a través del análisis de las identidades culturales colectivas en el largo plazo. Pero las conexiones entre el pasado y el presente no son un camino de ida sino una serie compleja de links dependiendo de las circunstancias y de los recursos de la comunidad, distinguiéndose tres tipos de relaciones de continuidad: cultura, recurrencia y reinterpretación. Su teoría es particularmente apta para analizar los orígenes de la nación armenia.

Palabras claves: Etnosimbolismo - nacionalismo - identidad - Armenia - Anthony D. Smith

\section{The etnosimbolism of Anthony D. Smith and the origins of Armenian nation}

\begin{abstract}
For Smith, one of the Ethno-symbolism's theorists, nations and nationalism can only be understood through the analysis of the collective cultural identities in the long run, but the conexions between the past and present are not a one way road but a series of complex links depending on the circumstances and the resources of the community. Three types of continuous relationships are identified: culture, recurrence and reinterpretation. His theory is particularly useful to analyze the origins of the Armenian nation.
\end{abstract}

Key words: Ethno-symbolism - nationalism - identity - Armenia - Anthony. D. Smith

TRABAJO RECIBIDO: 19/04/2018 TRABAJO ACEPTADO: 22/10/2018

\footnotetext{
* Doctor en Relaciones Internacionales, Universidad Nacional de Rosario (UNR, Argentina). Master of Business Administration por Dartmouth College. Correo electronic: rtorres1957@ @otmail.com
} 
Cuadernos de Política Exterior Argentina (Nueva Época), 128, diciembre 2018, pp. 51-66

ISSN 0326-7806 (edición impresa) - ISSN 1852-7213 (edición en línea)

\title{
Introducción
}

De las distintas teorías del nacionalismo que se han desarrollado en los últimos cincuenta años ${ }^{1}$ utilizaremos las teorías etnosimbolistas de Anthony D. Smith ${ }^{2}$ porque Smith es

\begin{abstract}
${ }^{1}$ Dentro de las teorías del nacionalismo hay cuatro grandes escuelas, primordialistas, perennialistas, modernistas y etnosimbolistas. Entre los primordialistas, que sostienen que las naciones son las divisiones naturales y primordiales de la humanidad y que el nacionalismo es universal, Edward Shils (1957) sostiene la importancia de los lazos primordiales basados en el lenguaje, religión, raza, etnicidad y territorio, Geertz (1963) re afirma el poder de los lazos primordiales atribuidos al givens (congruencias de sangre, lenguaje, costumbre, religión y territorio) de la existencia humana, givens que amenaza disolver los lazos civiles del estado moderno.
\end{abstract}

Entre los perennialistas, Adrian Hastings (1997) sostiene que naciones particulares han existido desde hace siglos.

Entre los modernistas, Kedouri (1960) sostiene que el nacionalismo es una doctrina inventada en Europa a comienzos del siglo XIX, y es una respuesta subversiva y revolucionaria a la alienación de los intelectuales alemanes y europeos, excluidos del poder por el absolutismo burocrático, Gellner (1964) sostiene que las naciones son creadas por el nacionalismo, que a su vez es la forma cultural asumida por la modernidad, o sea el moderno industrialismo. El nacionalismo se transforma en una forma cultural necesaria, una high culture. La definición de Gellner tiene bastante poca relación con las definiciones usuales de nacionalismo. Para Giddens (1985) es el estado moderno, centralizado, profesionalizado y territorializado que conjura al nacionalismo y genera la sensibilidad cultural de la soberanía. Mann menciona cuatro estadios en el desarrollo del nacionalismo en Europa, el primero de carácter religioso centrado en el siglo XVI, que incentiva networks de elite literaria, el segundo alrededor de 1700 con un crecimiento del alfabetismo debido a la expansión comercial y al militarismo estatal que producen una clase alta con el sentido de ciudadanía civil, el tercero de carácter decisivo, a partir de 1792, en que la crisis militar transforma las proto-nations en cross-classnations a través de la conscripción militar, impuestos de guerra y préstamos militares, lo que lleva a las clases propietarias a demandar una mayor representación política y politiza los conceptos de nación y pueblo, en la última fase a partir de fines del siglo XIX, el capitalismo industrial sustenta a las naciones a través de un estado en expansión, que asume mayores funciones, lo que lo hace más representativo, homogéneo y nacional y alienta un nacionalismo fanático y agresivo (1993). Para Breully (1993), el nacionalismo es un movimiento político y moderno y la política en el mundo moderno es sobre todo control sobre el estado, el nacionalismo es un argumento para alcanzar y retener ese control, su importancia está basada en su habilidad para ofrecer una plataforma común a las sub elites a través de la movilización, coordinación y legitimación de sus objetivos e intereses. Los movimientos nacionalistas, para Breully, tratan de unificar el estado, o renovarlo o más comúnmente, oponerse a un estado existente. Para Anderson (1991), el nacionalismo es una forma de discurso que imagina que la comunidad política es finita, soberana y horizontalmente cross-class. Las naciones se basan en las print communities vernáculas o sea los públicos lectores de novelas y periódicos en lenguas vernáculas, el crecimiento del público lector fue ayudado por la idea protestante de la lectura de la biblia en lenguas vernáculas y el crecimiento de las lenguas estatales, también para Anderson es importante una revolución en el concepto del tiempo, que ha pasado de ser mesiánico y cosmológico a ser linear y homogéneo. Para Anderson, la existencia de las naciones depende también de dos otras cuestiones, la diversidad global de las lenguas y la búsqueda de la inmortalidad. Para Hobsbawm (1983), las naciones y nacionalismo deben mucho a la tradición literaria y las creaciones historicistas de la historia nacional, la mitología y el simbolismo que florecieron en Europa a partir de 1830 y especialmente a partir de 1870. Antes de la primera guerra, hubo una notable proliferación de esas tradiciones inventadas (festivales nacionales, ceremonias por los caídos, himnos y banderas, eventos deportivos, etc). Estas tradiciones inventadas para Hobsbawm fueron creaciones de los ingenieros culturales que crearon símbolos, rituales, mitos, e historias para satisfacer las necesidades de las masas modernas movilizadas y politizadas por la industria y la democracia.

${ }^{2}$ Anthony D. Smith (1939-2016) fue un sociólogo británico, profesor de la London School of Economics and Political Science y es considerado uno de los fundadores de la disciplina de estudios sobre nacionalismo. Desde el punto de vista epistemológico sus posiciones han sido definidas como neoDurkhemian (Malesevic, 2007). En referencia a Smith, sólo se lo pueden entender como parte del proyecto de Durkheim ya que se inspira en la teoría general de la sociedad de Durkheim:

However, given that Smith awards primacy to a topic which is largely neglected in Durkheim's work, it is to be expected that he went much further than his predecessor. Being grounded in a Durkheimian ontology of the social world on one hand, and having to respond to non-and anti- 
el que mejor sintetiza el hecho de que la nación armenia, primero como ethnie y luego como nación no es un fenómeno moderno sino que existe desde la Antigüedad y es el que del punto de vista del etnosimbolismo mejor explica su evolución histórica al enfatizar su background étnico.

Smith, en The Ethnic Origins of Nations (1999), descarta las teorías modernistas y primordialistas para explicar el origen de las naciones. Smith descarta a los modernistas, en forma general, y sin entrar en detalle de las diferentes posturas, porque ven a la nación como un fenómeno moderno, producto estricto de desarrollos modernos como el capitalismo, la burocracia y el utilitarismo secular. También descarta a los primordialistas porque ven a las naciones y al nacionalismo como perennes, y naturales, prefiriendo Smith el estudio de las naciones contemporáneas en el contexto de su background étnico, basándose en la necesidad de un análisis que marque las diferencias y similitudes entre las modernas unidades nacionales y sus sentimientos y las unidades culturales y sentimientos de edades pretéritas, lo que luego identificará como etnosimbolismo (Smith, 1999:6-13).

Los etnosimbolistas tienen una serie de postulados que identifican a la teoría, a) la longue durée, b) pasado nacional, presente y futuro, o sea la relación de largo plazo entre el pasado nacional, el presente y el futuro, c) la base étnica de las naciones, que hacen que las comunidades étnicas o ethnies tengan los siguientes atributos 1. Un nombre identificatorio o un emblema, 2. Un mito común ancestral, 3. Memorias históricas y tradiciones compartidas, 4. Uno o más elementos de una cultura común, 5. Un link con un territorio histórico o homeland, 6. Un cierto grado de solidaridad entre las elites, d) el componente cultural de las ethnies, e) mitos y símbolos étnicos ${ }^{3}$ f) una historia étnica, g) los diferentes caminos para la formación de una nación y h) la longevidad del nacionalismo (Smith, 2009b:8-19).

Más específicamente, Smith en Ethno-symbolism and Nationalism (Smith, 2009a:23) argumenta que una de las preocupaciones de los etnosimbolistas es la necesidad de entender el mundo interior de la etnicidad y el nacionalismo a través del análisis de los elementos simbólicos y de sus dimensiones subjetivas. Smith cita la obra de John Armstrong, Nations before Nationalism (1982) y el argumento hecho por éste de que la persistencia de las identidades étnicas y de las naciones era más significativo que los cambios que las mismas sufren y que por eso, es necesario enfocarse en el límite simbólico que las separa de sus vecinos. Para Armstrong, la etnicidad debía entenderse de manera fenomenológica como un conjunto agrupado de actitudes cambiantes, sentimientos y percepciones, haciéndose necesario analizar la durabilidad de los grupos étnicos en términos de los mecanismos de límite simbólico como palabras, signos, lenguaje, vestimenta y arquitectura y la manera en que las elites comunican los símbolos y las estructuras míticas sucesivas en la que esos símbolos están incorporados. Mitos, símbolos y comunicación - el llamado complejo mito-simbólico dan la mejor herramienta conceptual para el análisis de grupos étnicos y naciones en la longue durée, como también para entender las funciones centrales de los mythomoteurs (conjunto de mitos constitutivos de una polity étnica) que garantizan la persistencia de las identidades étnicas en el tiempo. En The Ethnic Origin of Nations, Smith (1999) adopta el concepto de Armstrong de complejo mito-simbólico y se concentra en el rol de las ethnies en la formación de las naciones,

Durkhemian developments in contemporary sociology on the other hand (that is, neo-Marxist, neoWeberian and neo-Simmelian interpretations of nationalism), Smith had no choice but to go beyond the mere application of Durkheim's ideas to the study of nation formation. Instead his work is best read not merely as an attempt to take the Durkheimian position to its logical conclusion in the area of nations and nationalism, but also as a creative extension of this particular weltanschauung in its entirety. This is most pronounced in Smith's articulation of the ethnic origins of nations, his vision of nations as moral communities, and the notion of ethnic sacredness, all of which find their epistemological parallels in Durkheim's theories of solidarity, morality, religion, and collective consciousness. (Malesevic, 2007).

${ }^{3}$ Smith distingue varios tipos de mitos, a) los de origen temporal, b) los de locación o migración, c) de los ancestros, d) los de una era heroica o golden age, e) de decadencia y f) de regeneración (Smith, 2009b: 62-70). 
sosteniendo la necesidad de examinar los símbolos, mitos, memorias y valores individuales que componen los mythomoteurs que sostienen a las polities étnicas y la evolución histórica de las ethnies que forman la base de las futuras naciones (Smith, 2009a:24).

Para los etnosimbolistas, la realidad social es inconcebible fuera del simbolismo, consideran que los elementos culturales de símbolo, mito, memoria, valor, ritual y tradiciones son cruciales en el análisis de la etnicidad, las naciones y el nacionalismo, por el rol que han cumplido y continúan cumpliendo en modelar las estructuras sociales y las culturas, por el repertorio simbólico que cada uno de estos elementos culturales ha dado a cada comunidad que la hace diferente a otras comunidades y por último por el sentido de continuidad a lo largo del tiempo con las generaciones pasadas, sentimiento reforzado por la aceptación de los símbolos colectivos (Smith, 2009a:25).

Aquellos entienden que las relaciones étnicas son el factor individual más importante en el surgimiento, y la persistencia de las naciones y el nacionalismo ya que los diferentes tipos de lazos étnicos forman la base y el punto de partida para la creación de numerosas naciones y el sentido de relación étnica constituye muy a menudo un core étnico en una polity étnicamente heterogénea. Los lazos étnicos se presentan de distinta manera, en su forma más simple se puede hablar de categorías étnicas, la población es identificada por extranjeros como una categoría especial, sobre la base de algunos identificadores culturales como idioma, costumbres o religión pero sus miembros no tienen mitos ancestrales comunes y carecen de sentido de solidaridad. A veces tampoco se identifican por un nombre común. Las comunidades étnicas, que Smith llama ethnies son una forma más compleja y unificadora de etnicidad, ya que hablamos de una comunidad cuyas capas superiores tienen un sentido de solidaridad y cuyos miembros tienen un mito ancestral común que explica su origen común y su relación además de los identificadores culturales y las memorias compartidas. O sea, que para Smith, una ethnie es " a named and self defined human community whose members possess a myth of common ancestry, shared memories, one or more elements of common culture, including a link with a territory, and a measure of solidarity, at least among the upper strata" (Smith, 2009a:27).

Para Smith (Smith, 1999:22-32), las características de una ethnie son un nombre colectivo, un mito común de descendencia, una historia compartida, una cultura compartida distintiva, una asociación con un territorio específico y un sentido de solidaridad. Smith considera (Smith, 1999:32-40) que las bases para la formación de las ethnies son la sedentarización y la nostalgia, la religión organizada y la guerra entre los estados. Smith considera que en el centro de cada ethnie y de su peculiar etnocentrismo, entendido el etnocentrismo como la naturaleza general de los sentimientos de grupo pre modernos, hay dos tipos de mythomoteurs, los dinásticos y los comunales. Los primeros están ligados al soberano, su familia y dinastía, los segundos son alimentados por otras clases sociales y se enfocan en toda la comunidad y no solamente en una clase privilegiada. Estos últimos se encuentran en un amplio número de sistemas políticos: ciudades estados, confederaciones tribales, sectarias o diásporas. De los distintos tipos de etnocentrismo y sus característicos mythomoteurs, aquellos que contienen elementos sagrados y de fervor e imaginería religiosa son los más intensos y persistentes. Smith sostiene que hay dos tipos de ethnies, las aristocráticas laterales y las demóticas verticales. Las ethnies aristocráticas laterales tienen el sentido de etnicidad confinado principalmente a los nobles y clérigos, su limitada penetración social en ninguna forma disminuye el sentido de origen común y descendencia ni implica la rápida disolución de la misma. Las aristocráticas laterales persisten por lo general por varios siglos antes de disolverse o transformarse en ethnies demóticas e incipientes naciones. Las ethnies demóticas verticales enfatizan los lazos étnicos que las unen frente a los extranjeros o enemigos, tienen un énfasis marcado en la prohibición del sincretismo religioso, la asimilación cultural y los matrimonios con miembros de otras ethnies. Incluyen ciudades estados, ethnies fronterizas, confederaciones tribales, diásporas y sectas. Para Smith, son la ethnie antes que nación, etnicidad antes que nacionalidad y etnicismo definido como la forma de acción colectiva en defensa de la ethnie antes que nacionalismo las formas que caracterizan la vida social y cultural de la antigüedad y la 
Edad Media en Europa y el Cercano Oriente. Estas ethnies existieron dentro o separadas de las polities, o si se politizaron alcanzaron dominio sobre varias ethnies, aunque hay excepciones (casos en que ethnies y polities coincidieron) como por ejemplo el Antiguo Egipto (Smith, 1999:47-91). Smith presta especial atención a los factores que contribuyen a la disolución y supervivencia de las ethnies. Al analizar la disolución de las ethnies, menciona casos específicos, como la Antigua Fenicia que no logró diferenciarse de sus vecinos y que dependió culturalmente de otras culturas o el de Asiria, que muestra un alto grado de sincretismo religioso y de mezcla étnica. Al hablar de la supervivencia de las ethnies, Smith vuelve a recalcar que las aristocráticas laterales tienden a desaparecer al perder el poder político, especialmente cuando los mythomoteurs son fundamentalmente dinásticos. Las minorías étnicas que son verticales y demóticas y cuyos mythomotheurs son comunales tienden a sobrevivir si están en un territorio compacto, y cuando los sacerdotes y los textos sagrados ayudan a mantener la cohesión frente a la agresión externa. Las diásporas étnicas tienden a sobrevivir cuando el factor religioso está aún más marcado, como en el caso de los griegos ortodoxos, judíos y armenios (Smith, 1999:92-125).

Al resaltar el rol de la etnicidad en la formación y persistencia de las naciones y el nacionalismo, los etnosimbolistas no descartan otros factores económicos, sociales y políticos, como instituciones políticas, movilización de los ejércitos, actos de heroísmo, mitos de resistencia. Para Smith, es sobre la base del modelo étnico, y alrededor de una ethnie predominante que actores políticos ayudan a formar las naciones, como en los casos de Inglaterra y Francia en el período medieval y moderno, ya que sin un alto grado de homogeneidad cultural y étnica en el centro alcanzado a lo largo de varias generaciones, es dudoso que un estado fuerte se hubiera podido consolidar lo suficiente en dichos territorios para formar las futuras naciones y estados nacionales de Francia e Inglaterra.

Smith propone definir a la nación como "a named and self defining human community whose members cultivate shared memories, symbols, myths, traditions and values, inhabit and are attached to historic territories or homelands, create and disseminate a distinctive public culture and observe shared customs and standardised laws". Si bien esta definición se asemeja a la de ethnie, la diferencia está en la dimensión legal, territorial y política sobre la que coloca el énfasis (Smith, 2009a:28-30). Smith aclara que las ethnies se pueden encontrar junto a o dentro de las naciones y considera que las ethnies son el concepto más genérico, mientras que el de nación es el más específico, y aunque se haya originado en una ethnie previa es más inclusivo, más complejo y menos ligado a su base étnica original (Smith, 2010:14-15). Los estudios y los proyectos de la formación nacional no pueden restringirse a un solo período (moderno) así como el proceso de formación de varias naciones no puede estar ligado exclusivamente a la modernización, lo que hace necesario la referencia a las comunidades étnicas y al sentido de identidad étnica en períodos anteriores y a la relación entre ethnie y nación (Smith, 2009a:36). Para Smith, la principal tarea de los etnosimbolistas es realizar una historia cultural de la nación, o sea una investigación de las sucesivas self images y sentidos de identidad, de los conflictos ideológicos y los cambios sociales de una población culturalmente definida en un área o polity específica, lo cual permitirá describir los diferentes estadios de una historia de la nación y el nacionalismo. Comenzando por el juego cruzado de lazos étnicos, culturas y acción política, una historia cultural de la nación buscará primero las trazas de los procesos sociales y simbólicos en su formación, antes de distinguir los varios tipos de comunidad nacional y las diferentes rutas de la formación de la nación, así como su periodización (Smith, 2009a:39-40).

El nacionalismo, según Smith, puede ser definido como "an ideological movement for attaining and maintaining autonomy, unity and identity on behalf of a population deemed by some of its members to constitue and actual or potential nation" (Smith, 1991:73). Se trata de un movimiento activo, inspirado en la ideología y el simbolismo de la nación. El movimiento ideológico tiene varios aspectos, y una doctrina base con proposiciones sobre humanidad y política y prescripciones para la acción. Esta doctrina base sostiene que a) la humanidad está 
dividida en naciones, cada una con su historia, carácter y destino, b) la nación es la única fuente de poder político, c) la lealtad a la nación toma precedencia sobre cualquier otra lealtad, d) para ser libres los seres humanos deben pertenecer a una nación, e) las naciones requieren el máximo de autonomía y libre expresión y f) la paz y la justicia sólo se pueden construir sobre la base de una pluralidad de naciones libres. El nacionalismo es una doctrina sobre la nación, no sobre el estado, y si bien una nación libre muchas veces necesita un estado propio para la protección de su cultura, esto no es un requerimiento absoluto, tal cual lo demuestran naciones sin estado propio como Escocia, Quebec y Cataluña. Para los nacionalistas, las naciones son organismos naturales que existen en la naturaleza y son anteriores al estado, mientras que para los no nacionalistas, las naciones son descriptas como comunidades histórico culturales de prestigio y por lo tanto diferentes al estado, que puede ser definido como un conjunto de instituciones autónomas que ejercen un monopolio de coerción y extracción en un territorio dado. A pesar de las diferencias que algunos colocan entre patriotismo y nacionalismo, -el primero refiere al estado y el segundo a las comunidades étnicas-, para muchos estos tipos de lealtad son idénticos. A pesar de las diferencias entre patriotismo y nacionalismo para muchos autores, los nacionalismos tienen una serie de elementos comunes, autonomía, unidad, identidad, autenticidad, patria, dignidad, continuidad y destino, aunque la importancia de cada una de estas características varía según los nacionalismos y los períodos (Smith, 2009a:61-63).

El nacionalismo armenio surge a fines del siglo XIX bajo fuerte influencia europea (Smith, 2009b:218). Para Smith, modelos cívicos y territoriales de la nación tienden a producir nacionalismos anti coloniales antes de la independencia y nacionalismos integradores después de la independencia. Y los modelos étnicos y genealógicos de nación tienden a producir nacionalismo de secesión y diáspora antes de la independencia y nacionalismos irredentistas o "pan" luego de la independencia (Smith, 1991:82-83). "Nationalism is the secular modern equivalent of the pre modern, sacred myth of ethnic election" (Smith, 1991:84).

Para Smith, la formación de las naciones se debe principalmente a tres procesos, tres tipos de revolución que hacen primero su aparición en Occidente, aunque en largos períodos de tiempo y no siempre en el mismo lugar: la revolución en la esfera de la división del trabajo, la revolución en el control de la administración y la revolución en la coordinación cultural. Teniendo en cuenta que las tres revoluciones hicieron sentir sus efectos en diferentes momentos, en diferentes regiones y porque surgieron en situaciones sociales y culturales diferentes, las naciones que se fueron formando revelaron diferencias en el contenido y la forma. No sólo hubo naciones y nacionalismos religiosos y seculares, burgueses, aristocráticos y proletarios, conservadores y socialistas, burocráticos y populistas sino también dos formas y conceptos de nación, la territorial y la étnica El primer tipo surge en torno a un territorio y por los efectos de interacción dentro de límites geográficos definidos, con un claro marco legal, con una clara nacionalidad que lleva implícita la solidaridad y fraternidad y una cultura común. El segundo tipo de naciones surge en base a ethnies pre-existentes, por lo que se hace necesario transformar lazos étnicos en lazos y sentimientos nacionales a través de procesos de movilización, territorialización y politización. Estas dos concepciones de la nación moderna, la territorial y la étnica reflejan distintas experiencias históricas en el proceso de formación de las naciones. El territorialismo occidental lleva entre los siglos XIII y XVI a la formación de las polities étnicas de Inglaterra, Francia y Castilla que incorporan ethnies subordinadas a las ethnies dominantes. En Europa Central y Oriental y Medio Oriente, al llegar las revoluciones bastante más tarde, las polities existentes eran bastante diferentes, imperios multiétnicos, con una ethnie base que dominaba a las demás como en los imperios ruso, otomano y austríaco. En Europa Oriental y el Medio Oriente, los conceptos étnicos de nación jugaron un rol más importante aunque también se encuentran ideas territoriales. Para Smith, el proceso de formación de las naciones y el nacionalismo son conceptos occidentales, y el modelo occidental ejerce una clara influencia en todos los procesos subsiguientes. Según este autor, ninguna nación en proceso de formación puede sobrevivir sin una patria y mitos comunes como tampoco ninguna ethnie que aspira a ser una nación puede alcanzar sus objetivos sin alcanzar una división de trabajo y movilidad 
territorial y la igualdad de derechos y obligaciones de cada miembro, o sea ciudadanía (Smith, 1999:129-152).

Smith agrega dos definiciones conceptuales de gran importancia. Primero, la definición de estado nacional: "a state legitimated by the principles of nationalism, whose members possess a measure of nacional unity and integration (but not of cultural homogeneity)". Y segundo, la de identidad nacional: "the continuos reproduction and reinterpretation by the members of a national community of the pattern of symbols, values, myths, memories and traditions that compose the distinctive heritage of nations and the variable identification of individual members of that community with that heritage and its cultural elements" (Smith,

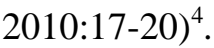

Para Smith, aunque el nacionalismo no determina qué grupos pueden convertirse en naciones ni porqué lo hacen, juega un rol importante en determinar cuando y donde las naciones surgirán ${ }^{5}$ (Smith, 1991:99).

De todas las identidades colectivas en el mundo de hoy, la identidad nacional es probablemente la más fundamental e inclusiva. El nacionalismo ha penetrado todos los rincones del planeta y al mismo tiempo, el mundo está dividido en estados nación y la identidad nacional sostiene en todos lados el camino de la soberanía popular y la democracia y a veces también el de la tiranía (Smith, 1991:143). El nacionalismo y la identidad nacional son fundamentales en el mundo de hoy, porque a) son globales, b) son generalizados (afectado la vida de individuos y comunidades) y c) debido a la complejidad y variedad de la nación y la identidad nacional (Smith, 1991:143-144). "Today national identity is the main form of collective identification. Whatever the feelings of individuals, it provides the dominant criterion of culture and idendity, the sole principle of government and the chief focus of social and economic activity" (Smith, 1991:170).

En Myths and Memories of the Nation, Smith se extiende sobre las diásporas particularmente, judíos, griegos y armenios, que constituyen lo que John Armstrong llama mobilized diasporas ${ }^{6}$. Smith les asigna a las archetypal diásporas, el concepto de chosenness, o

\footnotetext{
${ }^{4}$ Para Smith, identidad nacional y nación son "complex constructs" compuestos de componentes interrelacionados: étnicos, culturales, territoriales, económicos y político-legales. Conceptualmente para Smith, la nación combina dos tipos de dimensiones, una cívica y territorial y la otra étmica y genealógica, en proporciones diferentes según los casos. "It is this multidimensionality that has made national identity such a flexible and persistent force in modern life and politics, and allowed it to combine effectively with other powerful ideologies and movements without losing its character. We can illustrate this multifaceted power of national identity by looking at some of the functions it fulfils for groups and individual communities" (Smith, 1991:15). Estas funciones pueden ser divididas en externas e internas. Las externas son territoriales, económicas y políticas. La más importante función interna es la socialización de sus miembros como "nationals" y ciudadanos (Smith, 1991:16).

5 "In other words, what we mean by national identity comprises both a cultural and political identity and is located in a political community as well as a cultural one. This is significant because it means that any attempt to forge a national identity is also a political action with political consequences, like the need to redraw the geopolitical map or alter the composition of political regimes and states. Creating a world of nations has profound consequences for the global system of states as well as for individual states" (Smith, 1991:99).

${ }^{6}$ Armstrong (1976) define como diáspora a toda colectividad étnica que carece de base territorial dentro de una polity determinada, ie, es una minoría reducida a través de todas las porciones de la polity. Obviamente, la definición concierne a muchos tipos de diáspora, aunque Armstrong en su artículo de 1976 se refiere a dos, las diásporas proletarias y las diásporas movilizadas, y define a las movilizadas como grupos que sin tener ventajas de estatus, gozan de ventajas materiales y culturales dentro de polities multiétnicas, con respecto a otros grupos. $\mathrm{Y}$ a su vez subdivide a las movilizadas en dos grupos, archetypal y situational. La diferencia entre ambos es que la condición de diáspora es permanente en el primer grupo y temporaria en el segundo. Dentro del primer grupo él menciona a los judíos y armenios y en el segundo a los alemanes de Europa Oriental y a los chinos del Sudeste asiático aunque la distinción en referencia a una patria es más difícil de hacer, aún en el caso de judíos y armenios ya que en el primer
} 
sea un acto de Dios que identifica a un pueblo, el mito de la era dorada que antecede a la edad moderna y que forma parte de las memorias comunes de la ethnie y actúa como su mythomoteur, mitos afectados por experiencias negativas normalmente asociadas al trauma y la catástrofe y positivas, relacionadas con la provisión de canales de acción colectiva, situación que se da para Smith en los casos de los griegos ortodoxos, judíos y armenios (Smith, 2009b:203-224).

No deja lugar a dudas la existencia de la nación armenia en la Antigüedad ${ }^{7}$ y se la puede considerar el tipo ideal de nación, hablando de la Armenia del siglo V d. C (Smith, 2000:63).

caso se restableció una patria para los judíos en el Cercano Oriente en 1948 y en el segundo un estado propio en el siglo XX. La realidad es que la mayor parte de las diásporas cae en una situación intermedia. La gran diferenciación entre ambas diásporas se puede establecer en función del mito más que en realidades externas, más cercano al nacionalismo en el caso de la situational y de una profunda religiosidad en el caso de la archetypal.

${ }^{7}$ Para la mayoría de los especialistas armenios del siglo XIX y comienzos del siglo XX, la migración de los armenios desde sus tierras ancestrales (homeland de los indoeuropeos) al altiplano armenio ocurrió en la mitad del segundo milenio a. C., a través de los Balcanes y el Mar Egeo; también consideran que los urartianos, los habitantes originales del altiplano armenio no eran armenios. Los dos principales representantes de esta teoría son Suren Yeremian e Igor Diakonoff. Por el contrario, Rafael Ishkhanyan considera, a diferencia de los migracionistas, que los armenios son originarios del altiplano armenio (Geukjian, 2012:21; 23-24).

Yeremian sostuvo en 1974 que la confederación tribal de los hayasa-azzi (1500-1200 a. C, localizada en la parte superior del río Eufrates, en la región de las modernas ciudades de Erzinjan y Erzurum, en Turquía nororiental constituye el más antiguo componente étnico del pueblo armenio. Los hayasa-azzi hablaban una lengua hitita-luwian que tiene poco que ver con el armenio actual. Fueron los arimi-urumeans, una tribu original de Tracia-Frigia que llevó la lengua armenia desde los Balcanes hasta el altiplano armenio. Es en la región que se extiende desde el Monte Tauro hasta el Tauro armenio, cerca de las fuentes del río Tigris, incluyendo el valle del río Eufrates, donde se formó el núcleo del futuro estado armenio, ya que no podía ser subyugado ni por Urartu ni por Asiria. Los arimi-urumeans, que hablaban una lengua protoarmenia se mezclaron con los frigios e introdujeron la influencia de Tracia-Frigia en el armenio antiguo. Luego los armens contribuyeron junto a otras tribus a la caída del Reino de Urartu en el siglo VI a. C. y se establecieron en sus tierras, particularmente la región del lago Van, de esa forma formando el pueblo armenio (Geukjian, 2012:21-23).

Diakonoff, el otro teórico migracionista, en 1968 consideró que el elemento de origen en Tracia-Frigia no era el único componente del pueblo armenio. Diakonoff identificó a los mushki y a los urumeans como parte del grupo lingüístico proto-armenio que llegó al valle del Eufrates y al rio Arsanias en el segundo cuarto del siglo XII a. C, época de grandes migraciones. Los mismos se instalaron en las montañas Sasun y se encontraron con los pobladores originales, hurrian y luwian. Diakonoff fue de la opinión que en definitiva el pueblo armenio se formó en el valle del Eufrates por la mezcla de los hurrian, los luwian, y los proto-armenios (los mushki y los urumeans) y que el proceso comenzado en el siglo XII a. C se completó en el siglo VI a. C. (Geukjian, 2012:22-23).

Por el contrario, Ishkhanyan, en los años 1970s, escribió que los armenios eran el pueblo que hablaba la lengua armenia, dentro del grupo de lenguas indo-europeas y que era necesario ubicarlos históricamente cuando se convirtieron en un grupo separado dentro de las lenguas indo-europeas, aproximadamente entre el sexto y el cuarto milenio a. C. Ishkanyan localizó el territorio de los proto-europeos en la región oriental del Asia Menor (Anatolia Oriental) y partes de Irán en la región de los ríos Kura y Arax. En consecuencia, los armenios eran como los hititas y los luweans, los indo-iraníes y los griegos, una de las divisiones originales del grupo indo-europeo que se quedó en su proto-fatherland o no se movió de ella, y en consecuencia eran el pueblo originario del altiplano armenio y los urartianos deben ser considerados armenios (Geukjian, 2012:24-25).

Además de las teorías de los especialistas, existe la leyenda sobre los orígenes del pueblo armenio relatada por el historiador Movses Khorenatsi. Khorenatsi relata la leyenda de Hayk y su enemigo, Bel, el baal (dios o señor) asirio, que representa la opresión y a quien derrota fundando la nación armenia en la región del Monte Ararat. La lucha entre ambos intenta preservar la memoria de las guerras entre armenios y asirios. En la memoria colectiva, representa la lucha de un pueblo pequeño contra la tiranía de un gran imperio (Russell, 2004:32). 
Aquí es importante resaltar cuando una ethnie demótica, como Smith clasifica a los armenios, se transforma en nación (Smith, 1999:78) ${ }^{8}$ :

The important point here is that for an ethnie to become a nation, it must turn its members into citizens, but this does not entail having a state of one's own. One can obtain many of the rights of legal citizenzhip, without having outright independence, particularly in a federation. What is vital is turning a lateral ethnie into a cross class nation; and in the case of a demotic vertical ethnie, mobilizing the included membership for a common political goal. In other words, what matters for would be nation is inclusion and mobilization (Smith, 1999:166).

Para Steven Grosby, especialista del Antiguo Testamento:

Thus, speaking of Armenia in the fourth and fifth centuries of the common era, Grosby documents terms which suggest two referents of the collective self-consciousness constitutive of a nation of Armenia, a relation of a bounded areal jurisdiction, a territory, and a language... which was seen as common to Armenians in their territory. To this we may add a belief in the common ancestry of all Armenians (Grosby, 1991 citado por Smith, 2000:46-47).

Si bien se puede resaltar que los armenios constituían una nación ${ }^{9}$, aunque con los caveats del caso respecto a inclusión y movilización, ya en la antigüedad, cabe preguntarase qué sucedió cuando los armenios perdieron su territorio propio en forma definitiva (con la desaparición del reino armenio de Cilicia en 1375) y lo vieron ocupado por sucesivos invasores. Asimismo se debe tener en cuenta que como consecuencia de ello se fue desarrollando una diáspora importante manteniendo vinculaciones con sus terrirorios de origen. Por tanto surge la cuestión de si esa diáspora puede ser considerada una nación o se debe esperar al restablecimiento del estado armenio en 1918 para hablar de nación. Smith no tiene una respuesta clara al respecto (Smith, 2010:114-115) ${ }^{10}$.

Grosby analiza nuevamente el caso armenio en Biblical Ideas of Nationality (Grosby, 2002 citado por Smith, 2004:137; 139-141). Al convertirse al cristianismo en el 314 d. C., a través de la conversión de Trdat III y su familia, con un florecimiento religioso, artístico y cultural y un alfabeto e idioma propio, que sirven según Steven Grosby para acentuar la demarcación y la self-consciousness ${ }^{11}$ de la comunidad, la definición de lo armenio pasa de ser un concepto étnico y territorial a uno cultural. El énfasis se coloca en la propia versión del cristianismo armenio, en la constitución de una iglesia nacional autónoma y en su propio idioma reforzado por la creación de un alfabeto propio en el siglo V d. C. La actividad misionera de la

\footnotetext{
8 "Armenian feudal nobles, no longer able to rule an independent state, became more demotized, or rather the shared Armenian religio-ethnic culture was diffused down the social scale, until in the diaspora a more popular vertical community (or series of enclave communities) emerged in place of the earlier more aristocratic and lateral ethnie" (Smith, 1991:54).

9 "In these cases (Armenia and Israel), it makes sense to speak of nations in the ancient world, albeit cautiously and on a limited scale, marking the onset of a new pattern alongside that of the more common ethnic states. True, we cannot claim mass participation in politics, let alone full legal citizenship or economic unity, But, as we have seen a true application of this criteria would rule out many so called modern nations and delay the timing of the emergence of most nations, even in democracies, until after World War One "(Smith, 2010:118-119).

${ }^{10}$ Smith ve como intrigante los casos de Israel y Armenia que "We could also show the extent to which particular ethnies, such as the Armenians or Jews in late antiquity, approximated the national form of community, the subsequent shedding or loss of that national form and its re emergence or re acquisition in the modern period". Smith sostiene que las naciones son recurrentes pero no "continuous, features of human society and politics" (Smith, 2010:91).

${ }^{11}$ Smith pone el énfasis en que no hay que sobre enfatizar collective self affirmations, no sólo porque son definiciones voluntaristas sino también porque nos dicen poco de las colectividades culturales que se afirman como nación y que podrían incluir otro tipo de colectividades como ciudades estado o colectividades tribales, pero le da gran importancia a sus otras características como a "stable, bounded and translocal territory", único culto, dios, códigos de leyes, cultura pública, sistema de educación, y una serie de memorias, mitos y tradiciones propias de ese grupo (Smith, 2004:137-138).
} 
Iglesia Armenia en Georgia y la Albania Caucásica refuerza el rol de la iglesia. Tal como ha sido explicado por Nina Garsoian, la conversión al cristianismo empujó a Armenia hacia Roma y Occidente sin verse obligada a aceptar la posición del Concilio de Calcedonia en 451 d. C. El mito de la primera nación cristiana y la actividad misionera se convierten en una fuente de orgullo nacional. De igual importancia, es la derrota armenia en la batalla de Avarayr en $451 \mathrm{~d}$. C. A pesar de la derrota, el sacrificio del comandante armenio, Vardan Mamikonian y de muchos nobles ha sido conmemorado a lo largo de los siglos e inspirado a los armenios a la resistencia en la batalla y fue incorporado en las historias épicas escritas entre los siglos V y VIII d. C. Para el siglo $\mathrm{V}$ d. C., las elites armenias tenían una lectura providencial de su historia a través de mitos, símbolos y memorias, con sus tierras ancestrales, erkir Hayoc (la tierra de Armenia) con límites definidos en el centro de su propia comprensión. También recalca Grosby, la importancia de una cultura pública, con un único alfabeto y la institución de la iglesia con sus liturgias, clero y textos bíblicos que hizo que los armenios hicieran una alianza (Covenant) con Cristo para preservar el avrenk de los armenios, término que engloba no sólo la religión sino también las costumbres, leyes y tradiciones que caracterizaban a los armenios como armenios.

Para Smith, el concepto de la elección juega un rol esencial, fue un acto de Dios que eligió a una comunidad especial, para que viviera según sus leyes y recibiera sus bendiciones y esa elección se dio como recompensa por haber recibido la verdadera fe (Smith, 2009b:214) ${ }^{12}$.

Para los armenios, su época dorada, ligada a su sentido de destino colectivo (Smith, 2009b:264) se referencia en el reinado de Tigranes el Grande (95-55 a. C.), la dinastía de los Arshaguní o Arsacid en los siglos III y V d. C., y luego los Bagratuni o Bagratid en el siglo IX d. C. Son mitos que anteceden a la edad moderna y que funcionan como ${ }^{13}$ mythomoteurs y sus experiencias negativas o traumáticas se referencian no sólo en Avarayr sino en la pérdida de la independencia con el fin de los reinados de la dinastía Bagratuni o Bagratid y de los Lusignan en Cilicia, hasta las masacres de Abdul Hamid y el genocidio de 1915, sólo parcialmente recompensadas por la independencia y la victoria militar en la guerra de Nagorno-Karabagh (Smith, 2009b:215-217).

Para Smith, "where the community has been divorced from what it regards as its original habitat, as with Jews, Armenians, Greeks...the question of spatial origins looms especially large" (Smith, 2009b:64). El attachment de los armenios a su territorio y las pérdidas sufridas en Armenia Occidental durante el genocidio explican la posición armenia actual en NagornoKarabagh. O lo que es lo mismo:

for since ethnies are by definition associated with a given territory, not infrequently a chosen people with a particular sacred land, the presumed boundaries of the nation are largely determined by the myths and memories of the dominant ethnie, which include the foundation charter, the myth of the golden age and the associated territorial claims, or ethnic title deeds.

\footnotetext{
12 Para Smith, "the ideal of a covenant as the source of their ethnic election has given these communities of shared memory and origin myths a durability and self-renewing capacity which forms one of the bedrocks of their contemporary political struggles" (Smith, 2009b:267). También, el caso de los judíos, armenios y griegos es un claro ejemplo de estados étnicos que sobrevivieron en la historia a diferencia de los fenicios, nabateos y asirios para nombrar algunos. El rol central de las tradiciones religiosas y una clase sacerdotal diferenciada con sus ritos ayudan a mantener la identidad étnica a lo largo de los siglos (Smith, 1999:119).

13 Para Smith, los armenios de la diáspora vieron su época dorada en la era de Gregorio y Narses y en la primitiva iglesia apostólica cuyos ritos y liturgia han mantenido los armenios y cuya literatura sagrada ha inspirado el renacimiento de la era moderna:

In their sacred mythomoteur, Armenians saw themselves as a chosen people, their heroes as Old Testament warriors like Judas Maccabeus and their late Arsacid rulers (and Bagratid nobles) as of Jewish lineage; sharing the role of ancient Israel, the unique Gregorian-Armenian commmunity of the faithful stood out as the true repository of orthodoxy. Religion and ethnie converged to provide, as we shall see, a dynamic impetus to communal survival over the centuries and millennia (Smith, 1999:65).
} 
Hence the many conflicts, even today, for sundered parts of the ethnic homeland, in Armenia, in Kosovo, in Israel and Palestine, in the Ogaden, and elsewhere (Smith, 1991:39).

Shared memories of a rich ethno-history, and especially of golden ages, religious beliefs in ethnic election, and especially sacred covenants, and sentiments of belonging to ancestral homelands, especially sacred territories, these provide the sure foundations for nationalist movements aiming to regenerate the community or cultural category and form it into a modern nation in its ancestral homeland, preferably with its own protective state in a comity of national states (Smith, 2009b:271).

El renacimiento cultural armenio cuna del nacionalismo armenio en el siglo XIX tanto en la Armenia otomana como en la Armenia rusa como en las comunidades de la diáspora se explica en estos factores.

Smith confiere un rol importantísimo a los intelectuales en la consolidación del nacionalismo:

It is the intellectuals-poets, musicians, painters, sculptors, novelists, historians and archaeologists, playwrights, philologists, anthropologists and folklorists-who have proposed and elaborated the concepts and language of the nation and nationalism and have, through their musings and research, given voice to wider aspirations that they have conveyed in appropriate images, myths and symbols. The ideology and cultural core doctrines of nationalism may also be ascribed to social philosophers, orators and historians (Rousseau, Vico, Herder, Burke, Fichte, Mazzini, Michelet, Palacky, Karamzin) each elaborating elements fitted to the situation of the particular community for which he spoke ${ }^{14}$ (Smith, 1991:93) ${ }^{15}$.

14 "Under the aegis of different kinds of intelligentsia in conjunction with certain classes (usually bourgeoisie, but sometimes lower aristocracy or even workers) a new and distinctly national identity is created, which diffuses a reconstructed ethnic folk culture to all classes of the community. That identity has its civic elements, too; members are now legal citizens of the political ethno-nation, and they begin to define themselves in territorial terms as well. But the basis of their kind of national identity remains true to its demotic roots; the national identity created by intellectual s and intelligentsia among formerly vertical ethnies strives to stay close to its putative ethnic culture and boundaries. Mass mobilizing ethnic nationalism creates a political nation in the image of its presumed ethnic roots. Hence the very different form that national identities take among communities created by the mobilization and transformation of formerly demotic ethnies, at once more intense and often more inward looking than territorially based national identities" (Smith, 1999:129).

15 El renacer cultural armenio de fines del siglo XVII al siglo XIX se vivió por primera vez en las comunidades de la diáspora (Nalbandian, 1963:34-35).

En Europa, la congregación católica armenia Mekhitarista con sede en Venecia y luego también en Viena, fundada originalmente en Constantinopla, por Mekhitar de Sebaste (1676-1749) enriqueció la literatura armenia con numerosas publicaciones, literarias, artísticas, históricas, linguiísticas, arqueológicas y geográficas, entre las que destacan la publicación de una gramática en Armeno-Turkish, una gramática en grabar (armenio clásico) y un diccionario de la lengua armenia. Los mekhistaristas se aprovecharon de la pasión armenia por la traducción y en el siglo XIX, entre 1825 y 1850 tradujeron más de 130 volúmenes de obras literarias europeas, especialmente clásicos griegos y latinos y clásicos y obras modernas francesas e italianas (Oshagan, 2004:142-143).

En el siglo XIX, el debate sobre que lengua utilizar, clásico o vernáculo fue importante. A mediados de siglo el vernáculo se impuso, pero debido a la división de Armenia entre dos imperios, dos vernáculos emergieron, uno basado en el dialecto de Constantinopla y otro en el de Ereván. El armenio clásico sólo continuó como lengua favorita de una pequeña elite de intelectuales y el clero. Pero si bien, el vernáculo se impuso como lengua escrita, las masas con poca educación de las ciudades y los armenios de las provincias siguieron hablando o turco o varios formas dialectales que eran por lo general incompren sibles entre si (Panossian, 2006:133-134).

Entre los armenios occidentales, las reformas anunciadas por el sultán Abdul Mejid I en 1839 a través del Tanzimat, que prometían una mejora en la situación de los súbditos no musulmanes del Imperio Otomano, fomentaron el renacer cultural (Oshagan, 2004:151). 
Smith le otorga más importancia a la genealogía de las naciones que a la modernidad y la industrialización para determinar por qué y donde emerge el nacionalismo de un grupo y no el de otros (Smith, 1996:375-378; Panossian, 2006:273).

I am suggesting, then, that to understand modern nations and nationalism, we have to explore not only the processes and requirements of modernity, but also the genealogies of nations. In fact, we have to explore the impact of the processes of modernisation on those genealogies, and the way in which they give rise to selections and transformations by each generation of pre-existing ethnic ties and of the ethnic traditions they have inherited (Smith, 1996:377-378).

Smith le da un gran valor al sentido de identidad nacional ${ }^{16}$ y a las ideas de individualidad y autenticidad que él mismo expresa. Su definición que identifica dos relaciones, una entre los niveles individuales y colectivos de análisis ${ }^{17}$ y la otra entre la continuidad y el cambio de identidad que sostiene que las identidades y las comunidades culturales están sujetas

La contribución literaria a mediados del siglo XIX ayudó a crear un renacimiento literario que no se había visto en Armenia desde el siglo $\mathrm{V}$ d. C. El romanticismo fue la respuesta que encontraron los escritores para construir un nuevo nacionalismo que a su vez llevaría a la insurrección, particularmente entre los armenios orientales (Oshagan, 2004:164-165; Panossian, 2006:142).

Entre los armenios occidentales, la prensa adquirió gran relevancia y fue de gran ayuda para el desarrollo literario ya que casi tres cuartas partes de todos los textos producidos aparecieron en la prensa escrita. La mayoría de los escritores trabajaron o publicaron en un periódico; cerca de 300 publicaciones vieron la luz en el siglo XIX (Oshagan 2004:168-169). Entre los armenios occidentales, el género literario predominante fue el realista, especialmente luego de 1880, con temas que tenían que ver con la explotación, alienación y problemas sociales aunque también se desarrolla una literatura provincial, alejada del esnobismo de Constantinopla (Panossian, 2006:139-140).

Pero para entender mejor la situación de los armenios en el Imperio Otomano, es necesario hablar de su lucha por los derechos civiles y reformas administrativas, lo que se conoce internacionalmente como la "Cuestión Armenia" en la década de 1870. La mayor parte de los armenios en el Imperio Otomano eran campesinos, con sólo una minoría viviendo en los centros urbanos. En el transcurso del siglo XIX, la cambiante situación económica y política llevó a que muchos armenios fueran desposeídos de la tierra y tuvieran que instalarse en villas de emergencia en las ciudades en condiciones marginales. La "Cuestión Armenia" tenía características propias, la mayor parte de los cristianos del Imperio Otomano vivían en los Balcanes y estaban en proceso de separación del imperio, los árabes del imperio no habían sido colonizados por las tribus turcas, pero los 2 a 3 millones de armenios del Imperio Otomano vivían en todas las provincias. Había más armenios en Constantinopla que en cualquiera de las ciudades del altiplano armenio, también los armenios representaban una parte importante de la población de Cilicia y tenían un rol activo en la vida comercial de Anatolia Oriental. Si bien la mayoría de los armenios vivían en las provincias orientales del imperio (Armenia Occidental), sus tierras históricas, sede de los antiguos reinos armenios, ya no eran la mayoría en ese territorio. A lo largo de los siglos, las tribus turcas y los kurdos se habían establecido en las tierras ancestrales de los armenios y cuando se los combinaba, superaban a los armenios, por lo que los armenios eran el primer grupo en muchos distritos, pero sólo en algunos representaban la mayoría absoluta en Armenia Occidental (otomana). La distribución geográfica de los armenios en el imperio afectaba sus pretensiones políticas. Mientras los griegos, serbios, rumanos, búlgaros, y montenegrinos habían obtenido o estaban en vías de obtener su independencia, los armenios se contentaban en sus peticiones al gobierno otomano, en obtener un sistema de fiscalización directa, justicia civil y representación local que pusiera fin a las depredaciones de los funcionarios corruptos, terratenientes y tribus. Excepto por la rebelión de los montañeses de Zeytoun en 1862 contra el Imperio Otomano, hasta la década de 1870 los armenios otomanos no estaban dispuestos a defenderse (Hovannisian, 2004:203206).

${ }^{16}$ Identidad nacional junto a la autonomía nacional y unidad nacional son para Smith los tres objetivos del nacionalismo como ideología (Smith, 2010:28). Para Smith, "a sense of national identity however significant, is nevertheless a concomitant of the concept of the nation or national community: it sums up the members perceptions of difference and distinctiveness vis-à-vis other national communities and their members" (Smith, 2010:20).

${ }^{17}$ Smith sostiene que ambos niveles de análisis, el individual y el colectivo deben mantenerse separados (Smith, 2010:21). 
a procesos de cambio y disolución (Smith, 2010:20-22), lo que lleva en el caso armenio al cambio de los componentes de la identidad nacional a lo largo de los siglos, teniendo en cuenta las particularidades de la ethnie y nación armenia en cada momento histórico ${ }^{18}$. NagornoKarabagh fue el elemento catalizador que movilizó en 1988 a los armenios en Armenia y en la diáspora que entendieron que se presentaba una oportunidad histórica para su rescate como un primer paso hacia la recuperación de sus territorios históricos. Es como si después del genocidio y las pérdidas territoriales a comienzos del siglo XX, Karabagh fuera el elemento clave que le dio un nuevo significado a la identidad armenia. Nagorno-Karabagh fue y sigue siendo un claro indicativo de una marcha histórica de reunificación territorial y repatriación nacional que en el imaginario colectivo de la diáspora constituía la etapa posterior y lógica del reconocimiento del genocidio ${ }^{19}$. Nagorno-Karabagh y la independencia de Armenia en 1991 llevaron de hecho a modificar la idealización de la patria (ideal homeland), conceptualizada en la Causa Armenia o "Hay Tad" (Libaridian, 1999:82) al constatar la realidad de una lucha de liberación nacional en Nagorno-Karabagh y una Armenia independiente que necesitaban el apoyo de la diáspora. Se pasó de la temporalización de la Armenia Soviética como un museo y una patria temporaria hasta la reunificación con las tierras históricas de Armenia Occidental (Libaridian, 1999:124) a que fueran consideradas como el verdadero foco del pensamiento de la diáspora en referencia a la patria de los armenios. Se llegó al reconocimiento de una realidad sin abandonar el reclamo territorial que adquiere particular relevancia en virtud del centenario del genocidio.

Smith asigna una gran importancia al rol de una intelligentsia descontenta en cualquier agitación nacionalista. "The mobilization of the nation by the intellectuals was based on ancestral affiliations, emotions and cultural background" dándole al movimiento nacionalista legitimidad y definiendo a los miembros de una nación como una comunidad cultural (Smith, 1981:63-86 citado por Geukjian, 2012:137). Los intelectuales nacionalistas sostuvieron que la comunidad cultural armenia había sido el objeto de divisiones desde los años 1920 y que lo que se necesitaba era la preservación de la identidad armenia. La política de glasnost de Gorbachev de apertura a la crítica permitió a los intelectuales armenios liderar el movimiento de reunificación de la nación a partir de 1988 (Geukjian, 2012:137). Los líderes del Comité Karabagh crearon un sentido de interés común, incorporando símbolos de identidad compartida y de quejas. "Commitment to ethno-national demands was posible because the people's cultural identity and incentives for collective action was strong" (Geukjian, 2012:161).

O como dice Smith:

...When the new policies of perestroika and glasnost in the former Soviet Union brought into the open the national cleavages and ethnic aspirations in Eastern Europe and the Soviet republics. Starting even earlier in Poland, the tensions and conflicts, apparent from the mid 1990s in the Baltic states, the Ukraine, the Caucasus and Central Asia, helped to speed, even if they did not cause, the break up of the Soviet Union and its empire along ethno-national lines. One consequence of this new wave of ethnic nationalisms was to restore a measure of popular legitimacy to the aspirations of smaller peoples and subordinate nations, and more generally to nationalism, insofar as these popular movements appeared at this time to be

18 "This process of ethno-symbolic reconstruction involves the reselection, recombination and recodification of previously existing values, symbols, memories and the like, as well as the addition of new cultural elements by each generation" (Smith, 2010:22).

19 Para los años 70, el reconocimiento del Genocidio se transformó en la clave para entender la Causa Armenia. Los partidos políticos de la diáspora vincularon el reconocimiento del Genocidio al ideal de la Gran Armenia. Si bien el concepto de Causa Armenia fue usado por otros partidos de la diáspora, organizaciones e individuos, los Dashnaks se convirtieron en el portaestandarte de la Causa Armenia. El reconocimiento del Genocidio por parte de Turquía sería la base para el reclamo territorial en Armenia Occidental. El apoyo político provendría de los países que habían reconocido el Genocidio y de la URSS, que aceptaría una Armenia unida, sujeta al control soviético, aunque no independiente (Libaridian, 1999:82;128). La independencia de Armenia y Nagorno-Karabagh en 1991 cambió ese escenario. 
motivated by a genuine desire for collective freedom and democracy. Even if subsequent events in Eastern Europe, the former Soviet Union and, especially, the Balkans did not always bear out such hopes, and instead seemed to confirm an inextricable bond between an exclusive ethnic nationalism and neo-fascism, racism, and ethnic cleansing, the map of Europe had once more been largely recast along the lines of existing ethno-national cleavages, while East European politics had become increasingly bound up with the needs and interests of nationally defined states and their elites (Smith, 2010:130-131).

\section{Conclusiones}

Smith considera a Armenia el tipo ideal de nación, hablando de la Armenia del siglo V. d. C. y analiza como Armenia a la que clasifica como ethnie demótica se convierte en nación en sus territorios históricos independientemente de las teorías que uno sostenga para explicar el origen del pueblo armenio y su presencia en el altiplano armenio. En el caso de los armenios, la conversión temprana al cristianismo (Armenia fue el primer estado que adoptó el cristianismo oficialmente en el $301 \mathrm{~d}$. C., aunque probablemente el hecho tuviera lugar en el $314 \mathrm{~d}$. C.) y su imperio que perdió su independencia en $428 \mathrm{~d}$. C. (Era heroica), la batalla de Avarayr en $451 \mathrm{~d}$. C. (contra los persas), la resurrección del estado armenio con los Bagratids en 885-1045, el reino armenio de Cilicia (1198-1375), el comienzo de la diáspora, las masacres de Abdul Hamid (1894-1896) y el genocidio que comenzó en 1915 pueden funcionar como su mythomoteur, el mito constitutivo de la polity, que sostienen a lo largo de la historia a la ethnie y luego a la nación armenia. La existencia de un único alfabeto, creado a comienzos del siglo $\mathrm{V}$ d. C, y la Iglesia Apóstolica Armemia con sus ritos y tradiciones y su actividad misionera reforzó la alianza (covenant) de los armenios con Cristo para preservar el avrenk de los armenios y les ayuda a mantener su identidad a través de los siglos, cuando los armenios sufren invaciones y la ocupación de su territorio histórico y pierden un estado propio en el altiplano primero y luego en Cilicia.

Si bien siguiendo a Smith se puede considerar que los armenios constituían una nación ya en la antigüedad clásica, no está claro que sucede cuando los armenios pierden su último bastión independiente en Cilicia en 1375. La desaparición de éste, lleva al surgimiento de una diáspora importante que mantiene sus vínculos con los territorios históricos habitados por los armenios. Puede esa diáspora ser considerada como una nación, o al hablar de la nación armenia se debe incluir a los armenios en los territorios históricos y la diáspora o se debe esperar al establecimiento de un estado armenio en 1918. No hay respuestas fáciles al respecto.

La diáspora cumple un rol importante en el renacer cultural armenio a partir del siglo XVII. A partir del siglo XIX, será la intelligentsia de Constantinopla y Ereván la que lidere el renacimiento, que tiene por objetivo final la liberación de los armenios oprimidos por el Imperio Otomano en Armenia Occidental.

El genocidio es el mythomoteur clave del siglo XX, que domina el discurso de la diáspora. La intelligentsia vuelve a cumplir un rol clave en el movimiento Karabagh a partir de 1988 que culmina con la independencia de Armenia y Nagorno-Karabagh en 1991. Estos hechos modificaron la idealización de la patria armenia sostenida en el "Hay Tad" al reconocerse que la lucha por la liberación de Nagorno-Karabagh y Armenia misma necesitaban el apoyo de la diáspora. La diáspora, sin abandonar sus reclamaciones históricas en Armenia Occidental comenzó a ver a Armenia (la histórica Armenia Oriental) y a Nagorno-Karabagh como su verdadera patria. La pérdida de este último sería considerada por los armenios como un nuevo genocidio. 


\section{Bibliografía}

ANDERSON, B. (1991). Imagined Communities, Reflections on the Origins and the Spread of Nationalism, Second Edition, Verso, Londres

ARMSTRONG, J. (1976). Mobilized and Proletarian Diasporas en The American Political Science Review, Vol 70, N 2, Junio

ARMSTRONG, J. (1982). Nations before Nationalism, University of North Carolina Press, Chapel Hill, NC

BREUlLY, J. (1993). Nationalism and the State, Second Edition, Manchester University Press, Manchester

GEERTZ, C. (1963). The Integrative Revolution, en GEERTZ, C (ed), Old Societies and New States, Free Press, New York.

GELLNER, E. (1964). Thought and Change, Weidenfeld and Nicolson, Londres

GEUKJIAN, O. (2012). Ethnicity, Nationalism and Conflict in the South Caucasus, Nagorno-Karabakh and the Legacy of the Soviet Nationalities Policy, Ashgate, Farnham, Surrey

GIDDENS, A. (1985). The Nation State and Violence, Polity, Cambridge, Cambridgeshire.

GROSBY, S. (2002). Biblical Ideas of Nationality, Eisenbrauns, Warsaw, IN

GROSBY, S. (1991). Religion and Nationality in Antiquity en European Journal of Sociology 32, 229-65

HASTINGS, A. (1997). The Construction of Nationhood, Ethnicity, Religion and Nationalism, Cambridge University Press, Cambridge, Cambridgeshire

HOBSBAWM, E. and RANGER T. (eds) (1983). The Invention of Tradition, Cambridge University Press, Cambridge, Cambridgeshire

HOVANNISIAN, R. (2004). The Armenian Question in the Ottoman Empire 1876-1914 en

HOVANISSIAN, R. Armenian People from Ancient to Modern Times, Vol II, Foreign Dominion to Statehood, The Fifteenth Century to the Twentieth Century, St. Martin's Press, New York

KEDOURI, E. (1960). Nationalism, Hutchinson, Londres

LIBARIDIAN, G. (1999). The Challenge of Statehood, Armenian Political Thinking since Independence, Blue Crane Books, Watertown, MA

MALESEVIC, S. (2007). "Divine Ethnies" and "Sacred Nations", Anthony D. Smith and the neoDurkhemian Theory of Nationalism en Nationalism and Ethnic Politics, 10,4, 561-593

MANN, M. (1993). The Sources of Social Power, volume II, Cambridge University Press, Cambridge, Cambridgeshire

NALBANDIAN, L. (1963). The Armenian Revolutionary Movement, University of California Press, Berkeley

OSHAGAN, V. (2004). Modern Armenian Literature and Intellectual History from 1700 to 1915, en HOVANNISIAN, Richard, Armenian People from Ancient to Modern Times, Vol II, Foreign Dominion to Statehood, The Fifteenth Century to the Twentieth Century, St. Martin's Press, New York

PANOSSIAN, R. (2006). The Armenians, From Kings and Priests to Merchants and Commissars, C. Hurst, Londres

RUSSELL, J. (2004). The Formation of the Armenian Nation, en HOVANNISIAN, Richard, Armenian People from Ancient to Modern Times, Vol I, The Dynastic Periods: From Antiquity to the Fourteenth Century, St. Martin's Press, New York

SHILS, E. (1957). Primordial, personal, sacred and civil ties en British Journal of Sociology, Volume 8, Nro 2, págs 113-145, junio

SMITH, A. D. (2009a). Ethno-Symbolism and Nationalism. A Cultural Approach, Routledge, New York

SMITH, A. D. (2004). The Antiquity of Nations, Polity, Cambridge, Cambridgeshire 
SMITH, A. D. (1999). The Ethnic Origins of Nations, Blackwell, Oxford

SMITH, A. D. (2000). The Nation in History, Historiographical Debates about Ethnicity and Nationalism, University Press of New England, Hanover, $\mathrm{NH}$

SMITH, A. D. (2009b). Miths and Memories of the Nation, Oxford University Press, Oxford

SMITH, A. D. (2010). Nationalism, Polity, Cambridge, Cambridgeshire

SMITH, A. D. (1991). National Identity, Penguin, Londres

SMITH, A. D. (1996). Memory and Modernity, Reflections on Ernest Gellner's Theory of Nationalism en Nations and Nationalism, Volume 2, issue 3, 371-388, noviembre

SMITH, A. D. (1981). The Ethnic Revival, Cambridge University Press, Cambridge, Cambridgeshire 